RH-03-2002

\title{
A Note on Noncommutative Scalar Multisolitons
}

\author{
Bergfinnur Durhuus ${ }^{a}$ f and Thordur Jonsson ${ }^{b}$ ? \\ ${ }^{a}$ Matematisk Institut, Universitetsparken 5 \\ 2100 Copenhagen $\varnothing$, Denmark \\ ${ }^{b}$ University of Iceland, Dunhaga 3, \\ 107 Reykjavik, Iceland
}

\begin{abstract}
We prove that there do not exist multisoliton solutions of noncommutative scalar field theory in the Moyal plane which interpolate smoothly between $n$ overlapping solitons and $n$ solitons with an infinite separation.
\end{abstract}

\footnotetext{
${ }^{1}$ email: durhuus@math.ku.dk
}

${ }^{2}$ e-mail: thjons@raunvis.hi.is 
Solitons in scalar field theories were first studied in [1] in the limit of an infinite noncommutativity parameter $\theta$ where the kinetic term in the action can be neglected. The existence of rotationally invariant scalar noncommutative solitons at finite but sufficiently large $\theta$ was proved in [2] and stability of these solitons was established in [3]. In [4, 5] the moduli space of the infinite $\theta$ solitons is studied and it is argued that solitons attract at finite $\theta$ so that all multi-soliton solutions at finite $\theta$ are rotationally invariant around some point. In this paper we place this conjecture on a firm foundation. More precisely, we show that there does not exist a family of solitonic solutions interpolating smoothly between two overlapping solitons and two infinitely separated ones. The proof is based on using energy estimates which indeed show that the energy of two infinitely separated solitons is greater than that of two overlapping ones. Various aspects of the theory of solitons in noncommutative scalar field theories are discussed in [6, 47, 8, 9, 10, 11, 12, 13].

Solitons in a noncommutative two-dimensional scalar field theory with a potential $V$ are finite energy solutions to the variational equations of the energy functional

$$
\left.S(\varphi)=\operatorname{Tr}\left([a, \varphi]\left[\varphi, a^{*}\right]\right)+\theta V(\varphi)\right)
$$

where $a^{*}$ and $a$ are the usual raising and lowering operators of the simple harmonic oscillator and $\varphi$ is a self-adjoint operator on $L^{2}\left(\mathbf{R}^{2}\right)$. We assume as in [2, 3] that the potential $V$ is at least twice continuously differentiable with a second order zero at $x=0, V(x)>0$ if $x \neq 0$, and that $V$ has only one local minimum in addition to the one at $x=0$ located at $s>0$. For technical reasons we assume that the potential is analytic on a neighbourhood of the interval $[0, s]$ but we do not believe that this condition is necessary. We follow the notation of [2].

Let $\phi_{1}$ and and $\phi_{2}$ denote the stable one and two soliton solutions which were constructed for sufficiently large $\theta$ in [2, 3]. These solitons have the properties

$$
\phi_{1} \rightarrow s|0\rangle\langle 0|, \quad \phi_{2} \rightarrow s(|0\rangle\langle 0|+| 1\rangle\langle 1|)
$$

as $\theta \rightarrow \infty$, i.e., they converge to the infinite $\theta$ solitons of [1]. Let $U_{z}$ be the unitary operator on $L^{2}\left(\mathbf{R}^{2}\right)$ which implements the translation of the origin in the plane to the point $(x, y)$ and $z=x+i y$. Then $|z\rangle=U_{z}|0\rangle$ is a usual harmonic oscillator coherent state and the operator $s|z\rangle\langle z|$ is interpreted as an infinte $\theta$ soliton located 
at z. Similarly, one can argue [1] that

$$
\phi_{2, z}^{(\infty)}=s\left(\left|z_{+}\right\rangle\left\langle z_{+}|+| z_{-}\right\rangle\left\langle z_{-}\right|\right)
$$

where

$$
\left|z_{ \pm}\right\rangle=\frac{|z\rangle \pm|0\rangle}{\sqrt{2\left(1 \pm e^{-|z|^{2} / 2}\right)}}
$$

is a two soliton solution at infinte $\theta$ with the solitons located at $z$ and the origin. Clearly

$$
\left(\phi_{2, z}^{(\infty)}-s(|z\rangle\langle z|+s| 0\rangle\langle 0|) \rightarrow 0 \quad \text { as }|z| \rightarrow \infty\right.
$$

and

$$
\phi_{2, z}^{(\infty)} \rightarrow s(|0\rangle\langle 0|+| 1\rangle\langle 1|) \quad \text { as }|z| \rightarrow 0
$$

It is natural to interpret the operator

$$
\phi_{1, z}=U_{z} \phi_{1} U_{z}^{*}
$$

as the finite $\theta$ one soliton solution located at $z$. The question is whether there is a finite $\theta$ analogue of $\phi_{2, z}^{(\infty)}$. In [4] it is argued that this is not the case. The argument is based on computing the energy of the infinite $\theta$ solution using the finite $\theta$ energy functional and observing that the energy depends on the separation between the solitons. Here we provide a proof.

Let us assume that there exists a family of solitons, $\phi_{2, x}, x \geq 0$, depending smoothly on $x$, such that $\phi_{2,0}=\phi_{2}$ and

$$
\lim _{x \rightarrow \infty}\left(\phi_{2, x}-\phi_{1, x}-\phi_{1}\right)=0
$$

where the convergence above is in the norm $\|\cdot\|_{2,2}$ defined by

$$
\|\phi\|_{2,2}^{2}=\operatorname{Tr}\left([a, \phi]\left[\phi, a^{*}\right]+\phi^{2}\right) .
$$

Then the energy $E(x)=S\left(\phi_{2, x}\right)$ is a differentiable function of $x$ and since $\phi_{2, x}$ is a critical point of $S$ we have $E^{\prime}(x)=0$ so $E(x)$ is a constant. Hence,

$$
S\left(\phi_{2}\right)=E(0)=\lim _{x \rightarrow \infty} E(x)=2 S\left(\phi_{1}\right)
$$

In order to prove the last equality in (10) we write

$$
S(\phi)=K(\phi)+\theta \operatorname{Tr} V(\phi)
$$


where

$$
K(\phi)=\operatorname{Tr}\left([a, \phi]\left[\phi, a^{*}\right] .\right.
$$

We first show that

$$
K\left(\phi_{2, x}\right) \rightarrow 2 K\left(\phi_{1}\right)
$$

as $x \rightarrow \infty$. By the triangle inequality

$$
\begin{aligned}
\left|K\left(\phi_{2, x}\right)^{1 / 2}-K\left(\phi_{1, x}+\phi_{1}\right)^{1 / 2}\right| & \leq K\left(\phi_{2, x}-\phi_{1, x}-\phi_{1}\right)^{1 / 2} \\
& \leq\left\|\phi_{2, x}-\phi_{1, x}-\phi_{1}\right\|_{2,2} .
\end{aligned}
$$

In order to establish (13) it therefore suffices to show that $K\left(\phi_{1, x}+\phi_{1}\right) \rightarrow 2 K\left(\phi_{1}\right)$ as $x \rightarrow \infty$. We have

$$
\begin{aligned}
K\left(\phi_{1, x}+\phi_{1}\right) & =K\left(\phi_{1, x}\right)+K\left(\phi_{1}\right)+2 \operatorname{Re} \operatorname{Tr}\left[a, \phi_{1, x}\right]\left[\phi_{1}, a\right] \\
& =2 K\left(\phi_{1}\right)+2 \operatorname{Re} \operatorname{Tr}\left[a, \phi_{1, x}\right]\left[\phi_{1}, a\right] .
\end{aligned}
$$

If $f$ is the function on $\mathbf{R}^{2}$ which corresponds to $\phi_{1}$ under Weyl quantization, then

$$
\operatorname{Tr}\left[a, \phi_{1, x}\right]\left[\phi_{1}, a\right]=\frac{1}{4 \pi} \int_{\mathbf{R}^{2}} \partial_{\bar{z}} f(z-x) \partial_{z} \bar{f}(z) d z d \bar{z}
$$

and the last integral tends to zero as $x \rightarrow \infty$ by the Riemann-Lebesgue Lemma (this can also be seen directly since $f$ is a gaussian).

We next prove the convergence

$$
V\left(\phi_{2, x}\right) \rightarrow 2 V\left(\phi_{1}\right)
$$

as $x \rightarrow \infty$ for $V$ a polynomial. The proof generalizes without difficulty to the case where $V$ is analytic on a neighbourhood of $[0, s]$ by means of the analytic functional calculus. Let $\|\cdot\|_{2}$ denote the usual Hilbert-Schmidt norm. For $n \geq 2$ we have

$$
\begin{aligned}
\left|\operatorname{Tr} \phi_{2, x}^{n}-\operatorname{Tr}\left(\phi_{1, x}+\phi_{1}\right)^{n}\right| & =\operatorname{Tr}\left(\sum_{k=0}^{n-1} \phi_{2, x}^{k}\left(\phi_{2, x}-\phi_{1, x}-\phi_{1}\right)\left(\phi_{1, x}+\phi_{1}\right)^{n-1-k}\right) \\
& \leq \sum_{k=0}^{n-1}\left\|\phi_{2, x}-\phi_{1, x}-\phi_{1}\right\|_{2}\left\|\phi_{2, x}\right\|_{2}^{k}\left\|\phi_{1, x}+\phi_{1}\right\|_{2}^{n-1-k} \\
& \leq c\left\|\phi_{2, x}-\phi_{1, x}-\phi_{1}\right\|_{2}
\end{aligned}
$$

for an $x$-indpendent constant $c$ since

$$
\left\|\phi_{x, 1}+\phi_{1}\right\|_{2} \leq\left\|\phi_{1, x}\right\|_{2}+\left\|\phi_{1}\right\|_{2}=2\left\|\phi_{1}\right\|_{2} .
$$


On the other hand,

$$
\operatorname{Tr}\left(\phi_{1, x}+\phi_{1}\right)^{n} \rightarrow 2 \operatorname{Tr} \phi_{1}^{n}
$$

as $x \rightarrow \infty$ since all cross terms between $\phi_{1, x}$ and $\phi_{1}$ vanish by the Riemann-Lebesgue Lemma as before. We have therefore established the convergence (17) for any polynomial $V$ with a second order zero at the origin and this completes our proof of (10). Now we return to our main line of argument.

Differentiating the equation $S\left(\phi_{2}\right)=2 S\left(\phi_{1}\right)$ with respect to $\theta$ we obtain

$$
\operatorname{Tr} V\left(\phi_{2}\right)=2 \operatorname{Tr} V\left(\phi_{1}\right) .
$$

We claim that the above equality is violated for all sufficiently large values of $\theta$ and therefore the family $\phi_{2, x}$ does not exist for large values of $\theta$.

Let $\lambda_{0}^{(1)}, \lambda_{1}^{(1)}, \lambda_{2}^{(1)}, \ldots$ and $\lambda_{0}^{(2)}, \lambda_{1}^{(2)}, \lambda_{2}^{(2)}, \ldots$ denote the eigenvalues of $\phi_{1}$ and $\phi_{2}$ in the harmonic oscillator basis. Then the eigenvalues form decreasing sequences of positive numbers and in [3] it is proven that

$$
\begin{aligned}
& \lambda_{0}^{(1)}=s-\frac{2 s}{\theta V^{\prime \prime}(s)}+O\left(\theta^{-2}\right) \\
& \lambda_{1}^{(1)}=\frac{2 s}{\theta V^{\prime \prime}(0)}+O\left(\theta^{-2}\right) \\
& \lambda_{0}^{(2)}=s-O\left(\theta^{-2}\right) \\
& \lambda_{1}^{(2)}=s-\frac{4 s}{\theta V^{\prime \prime}(s)}+O\left(\theta^{-2}\right) \\
& \lambda_{2}^{(2)}=\frac{4 s}{\theta V^{\prime \prime}(0)}+O\left(\theta^{-2}\right)
\end{aligned}
$$

and all the other eigenvalues are $O\left(\theta^{-2}\right)$. It follows that

$$
\begin{aligned}
& \operatorname{Tr} V\left(\phi_{1}\right)=V\left(\lambda_{0}^{(1)}\right)+V\left(\lambda_{1}^{(1)}\right)+\sum_{n=2}^{\infty} V\left(\lambda_{n}^{(1)}\right) \\
& =V(s)+\left(\frac{2 s^{2}}{V^{\prime \prime}(s)}+\frac{2 s^{2}}{V^{\prime \prime}(0)}\right) \frac{1}{\theta^{2}}+\sum_{n=2}^{\infty} V\left(\lambda_{n}^{(1)}\right)+O\left(\theta^{-3}\right)(28)
\end{aligned}
$$

Similarly,

$$
\begin{aligned}
& \operatorname{Tr} V\left(\phi_{2}\right)=V\left(\lambda_{0}^{(2)}\right)+V\left(\lambda_{1}^{(2)}\right)+V\left(\lambda_{2}^{(2)}\right)+\sum_{n=3}^{\infty} V\left(\lambda_{n}^{(2)}\right) \\
& =2 V(s)+\left(\frac{8 s^{2}}{V^{\prime \prime}(s)}+\frac{8 s^{2}}{V^{\prime \prime}(0)}\right) \frac{1}{\theta^{2}}+\sum_{n=3}^{\infty} V\left(\lambda_{n}^{(2)}\right)+O\left(\theta^{-3}\right)
\end{aligned}
$$


In order to estimate the tails of the sums we use the following argument. There is a number $b \in(0, s)$ such that $V(x) \leq x V^{\prime}(x)$ for $0<x<b$. Taking $\theta$ sufficiently large so that $\lambda_{2}^{(1)}<b$ and $\lambda_{3}^{(2)}<b$ we see that

$$
\begin{aligned}
\sum_{n=2}^{\infty} V\left(\lambda_{n}^{(1)}\right) & \leq \sum_{n=2}^{\infty} \lambda_{n}^{(1)} V^{\prime}\left(\lambda_{n}^{(1)}\right) \\
& \leq \lambda_{2}^{(1)} \sum_{n=2}^{\infty} V^{\prime}\left(\lambda_{n}^{(1)}\right) \\
& \leq c \theta^{-2} \sum_{n=2}^{\infty} V^{\prime}\left(\lambda_{n}^{(1)}\right)
\end{aligned}
$$

where $c$ is a constant.

Similarly,

$$
\sum_{n=3}^{\infty} V\left(\lambda_{n}^{(2)}\right) \leq c \theta^{-2} \sum_{n=3}^{\infty} V^{\prime}\left(\lambda_{n}^{(2)}\right)
$$

We also have (see [2, 3])

$$
\sum_{n=0}^{\infty} V^{\prime}\left(\lambda_{n}^{(1)}\right)=\sum_{n=0}^{\infty} V^{\prime}\left(\lambda_{n}^{(2)}\right)=0
$$

SO

$$
\sum_{n=2}^{\infty} V^{\prime}\left(\lambda_{n}^{(1)}\right)=-V^{\prime}\left(\lambda_{0}^{(1)}\right)-V^{\prime}\left(\lambda_{1}^{(1)}\right)=O\left(\theta^{-2}\right)
$$

and

$$
\sum_{n=3}^{\infty} V^{\prime}\left(\lambda_{n}^{(2))}=-V^{\prime}\left(\lambda_{0}^{(2)}\right)-V^{\prime}\left(\lambda_{1}^{(2)}\right)-V^{\prime}\left(\lambda_{2}^{(2)}\right)=O\left(\theta^{-2}\right) .\right.
$$

It follows that the tails of the sums in (28) and (30) are $O\left(\theta^{-4}\right)$ and Eq. (21) can be written as

$$
\left(\frac{1}{V^{\prime \prime}(s)}+\frac{1}{V^{\prime \prime}(0)}\right) \frac{4 s^{2}}{\theta^{2}}+O\left(\theta^{-3}\right)=\left(\frac{1}{V^{\prime \prime}(s)}+\frac{1}{V^{\prime \prime}(0)}\right) \frac{8 s^{2}}{\theta^{2}}+O\left(\theta^{-3}\right)
$$

which is impossible for large values of $\theta$.

By a similar argument one can rule out the existence of a multisoliton solution $\phi_{n, z_{1}, \ldots, z_{n}}$ at large but finite $\theta$ with the properties

$$
\phi_{n, z_{1}, \ldots, z_{n}} \sim s \sum_{i=0}^{n-1}|i\rangle\langle i|, \quad z_{i} \rightarrow 0
$$

and

$$
\phi_{n, z_{1}, \ldots, z_{n}} \sim \sum_{i=1}^{n} \phi_{1, z_{i}}, \quad\left|z_{i}-z_{j}\right| \gg 1, i \neq j
$$


The fact that general multisolitons do not exist as solutions to the static equations of motion at finte $\theta$ will most likely make it more difficult to establish rigorously the results about moduli space approximation [5]. To actually control such approximations seems to require an analysis of the time dependent equations of motion.

Acknowledgements. The work of B. D. is supported in part by MaPhySto funded by the Danish National Research Foundation. This research was partly supported by TMR grant no. HPRN-CT-1999-00161. T. J. is indebted to the Niels Bohr Institute for hospitality and would like to thank J. Ambjørn, P. Austing and L. Thorlacius for discussions.

\section{References}

[1] R. Gopakumar, S. Minwalla and A. Strominger, Noncommutative solitons, JHEP 0005020 (2000) hep-th/0003160

[2] B. Durhuus, T. Jonsson and R. Nest, Noncommutative scalar solitons: existence and nonexistence Phys. Lett. B 500 (2001) 320 [hep-th/0011139]

[3] B. Durhuus, T. Jonsson and R. Nest, The existence and stability of noncommutative scalar solitons, hep-th/0107121

[4] R. Gopakumar, M. Headrick and M Spradlin, On noncommutative multisolitons, hep-th/0103256

[5] L. Hadasz, U. Lindstrøm, M. Rocek and R. von Unge, Noncommutative multisolitons: moduli spaces, quantization, finite $\theta$ effects and stability, JHEP 0106 (2001) 040 hep-th/0104017

[6] C.-G. Zhou, Noncommutative scalar solitons at finite $\theta$, hep-th/0007255

[7] A. S. Gorsky, Y. M. Makeenko and K. G. Selivanov, On noncommutative vacua and noncommutative solitons, Phys.Lett. B492 (2000) 344-348 [hep-th/0007247]

[8] U. Lindstrøm, M. Rocek and R. von Unge, Non-commutative soliton scattering, JHEP 0012 (2000) 004 hep-th/0008108 
[9] A. Solovyov, On noncommutative solitons, Mod.Phys.Lett. A15 (2000) 22052218 hep-th/0008199

[10] D. Bak and K. Lee, Elongation of moving noncommutative solitons, Phys.Lett. B495 (2000) 231-236 [hep-th/0007107]

[11] Y. Matsuo, Toplogical charges of noncommutative soliton, Phys.Lett. B499 (2001) 223-228 hep-th/0009002

[12] M. G. Jackson, The stability of noncommutative scalar solitons, JHEP 0109 (2001) 004 hep-th/0103217

[13] M. Spradlin and A. Volovich, Noncommutative solitons on Kähler manifolds, hep-th/0106180 